\title{
Health utility indices and equity considerations
}

\author{
Han Bleichrodt \\ iMTA, Department of Health Policy and Management, Erasmus Unilersity, PO Box 1738, 3000 DR, \\ Rotterdam. The Netherlands
}

Received 1 October 1994: accepted 1 May 1996

\begin{abstract}
The aim of this paper is to propose methods that incorporate equity concerns into cost utility analysis. The focus of the paper is on QALYs, but the results apply to health utility indices in general. Two interpretations of QALYs are considered: QALYs as (von Neumann Morgenstern) utilities and QALYs as measures of health. A justification is provided for aggregating consistently scaled "QALYs as utilities" over individuals. The conditions underlying unweighted aggregation of QALYs are identified. These conditions exclude two common types of equity concern. Algorithms are proposed that take into account equity concerns and that are relatively easy to apply.
\end{abstract}

JEL classification: 110

Keynords: Equity: Uitity theory: Social choice theory: Health; Cost utility analysis

\section{Introduction}

Utility indices for health care programmes, such as QALYs, have been criticized for being primarily concerned with efficiency, ignoring equity implications (see, for example. Lockwood (1988); Harris (1988); Smith (1987); Broome (1988); Broome (1993)). The importance of incorporating equity considerations into cost utility analysis has been widely acknowledged by researchers in the field (e.g. Williams, 1993). However, despite statements of intent, few attempts have been made thus far to actually develop methods by means of which equity considerations can be taken into account in cost utility analysis. One of the few 
exceptions is Wagstaff (1991), who suggested combining equity and efficiency considerations in cost utility analysis by means of the social welfare function underlying Atkinson's index of inequality (Atkinson, 1970). However, Wagstaff did not pursue this idea any further, and in particular did not indicate how the parameters of this social welfare function can be assessed by experimental methods.

The aim of this paper is to derive functional forms that allow trading off the efficiency gains of a health care programme against their equity implications. Given that QALYs are the most frequently used outcome measure in cost utility analysis, we will refer to the gains of a health care programme as the number of QALYs gained. However, it should be emphasized here that all results derived in the sequel of the paper apply to other utility-based outcome measures as well. It is also important to realize that this paper is concerned with equity concerns over consistently scaled QALYs, consistent in the sense that QALYs are comparable over individuals. This distinguishes this paper for example from the paper by Gafni and Birch (1991), in which the influence of equity considerations on the scaling of the von Neumann Morgenstern utility function is shown and in which aigorithms are developed to ensure consistent scaling. However, the two approaches are not completely indepetiuent. We briefly return to this issue in Section 6.

The functional derivations presented in this paper are based on the tools of multi-aiuribute utility theory. Multi-attribute utility theory has been developed as a procedure to make explicit the trade-off between conflicting objectives. Two interpretations of QALYs that have been distinguished in the literature are considered: QALYs as von Neumann Morgenstern (vNM) utilities and QALYs as measures of health. 'It has been claimed that the QALYs as utilities approach lacks a theoretical foundation. since utilities cannot be interpersonally compared in a meaningful way. This problem is addressed in Sections 3 and 4.

The structure of the paper is as follows. Section 2 discusses the two interpretations of QALYs. Sections 3 and 4 provide a rationale for aggregating consistently scaled "QALYs as utilities" over individuals. In Section 3 it is argued that if we want to incorporate equity considerations in cost utility analysis. full interpersonal comparability of utilities is required. In Section 4 an argument is presented that vNM utilities can meaningfully be interpersonally compared. in Section 5 the conditions are identified under which the aggregation of QALYs over individuals takes the form of "QALY-utilitarianism," i.e. the unweighted summation of QALYs over individuals. Section 6 shows that these conditions inhibit the inclusion of two common types of equity concern: a concern for the fairness of the allocation process, generally referred to as ex ante equity, and a concern for the

These wo interpretations are not necessarily mutually exclusive. 
(final) distributional implications, referred to as ex post equity. Replacing the relevant conditions by alternative conditions allows the inclusion of ex ante and ex post equity concerns. In Sections 7 and 8 three procedures are proposed by means of which equity concerns can be captured in cost utility analysis. The procedures described in Section 7 only address ex post equity. In Section 8 a procedure is described that simultaneously takes into account ex ante equity and ex post equity. Section 9 contains a summary and discussion of the main findings of the paper. Appendix A contains proofs of results presented in the main text of the paper.

\section{Interpretations of QALYs}

The definition of the number of QALYs for an individual, as given by Pliskin et al. (1980), is the following: ${ }^{2}$

$$
Q A L Y=\sum_{t=1}^{T} u\left(q_{t}\right)
$$

where $T$ stands for the number of periods remaining the individual still has to live, $q_{t}$ stands for the quality of life level attained in period $t$ and $u\left(q_{t}\right)$ is the utility of living in health state $g_{i}$ at period $t$. Ai least two interpretations have been distinguished in tie literature ${ }^{3}$ as to what the number of QALYs represents QALYs as vNM utilities and QALYs as measures of health. According to Torrance (1986): "In one approach health state utilities are ciaimed to be utilities obeying the axioms of von Neumann Morgenstern utility theory...In the otlis: approach...health state utilities are claimed to measure the overall quality of lif:" (p. 27).

With respect to the first interpretation, QALYs as vNM utilities, conditions have to be imposed on the individual preference relation to ensure that a QALY is a valid vNM utility. Criticism that decision making based on QALYs may not accurately reflect individual preferences is based on the presumption that ideally a QALY should be a vNM utility. In the interpretation of QALYs as vNM utilities, we abstract from the discussion whether the conditions that equate QALYs and VNM utilities are reasonable and it is simply assumed that the individual preference relations satisfy these conditions (for a critical evaluation of these condition see, for example, Mehrez and Gafni (1989); Loomes and McKenzie (1989)).

\footnotetext{
"One may object against this formulation in that it is unnecessarily simple and that. for eximple. discounting should be allowed for. However, this simple representation does not imply a loss of generality in terms of the results of this paper: all results carry over straightforwardly if a more general expression is substituted.

${ }^{3}$ Nord (1994) provides a third interpretation: QAL.Ys as a social value. We will not consider this interpretation for the obvious reason that in this interpretation aggregation plays no rols.
} 
However. it should be noted that if the preference conditions underiying the QALY measure are not satisfied, another utility-based outcome measure can simply be substituted. As remarked already in the Introduction, all results presented in the remainder of the paper apply to other utility-based measures as well.

The second interoretation, QALYs as measures of health, is rooted in the extra-welfarist tradition which originates from Sen (1979) and which has been applied to health by Culyer (1989). Wagstaff (1991) states the following: "Though utility theory is frequently used in the derivation of quality of life scores, it is used simply to measure people's health rather than the utility they derive from it" (p. 23). Part of the appeal of this latter approach stems from the fact that the comparability of "QALYs as utilities" across individuals may be problematic.

It is not an aim of this paper to decide which of these two interpretations is most appropriate. The equity algorithms presented in Sections 7 and 8 have been developed with the intention of being applicable under both interpretations. However. equity considerations relate to comparisons between individuals and therefore it has to be established first whether QALYs can be aggregated in both interpretations. The common way to aggregate QALYs is by unweighted summation. Because of this, the QALY approach has been criticized as embodying a return to classical, or Benthamite, utilitarianism. Wagstaff (1991) has argued that in the interpretation of QALYs as measures of health this criticism does not stand scrutiny. Classical utilitarianism focuses on the aggregation of utilities wh eas the QALYs as measure of health approach mainly sees QALYs as reflecting characteristics of people without being concerned with the utility they derive from these characteristics. The idea behind this line of argument is that characteristics do not face problems of measurability and comparability across individuals. In the temainder of this paper this view is taken for granted. It is assumed that in the interpretation as a health measure, QALYs can indeed be aggregated across individuals and that the equity algorithms to be developed later can be applied to QALYs as measures of health.

The assertion made in this paper that QALYs as vNM utilities can also be meaningfuliy aggregated requires clarification. The question whether vNM utilities are interpersonally comparable and do have a meaning in social welfare analysis has provoked much debare over the past five decades. In the next section we will establish that to incorporate equity considerations into cost utility analysis full interpersonal comparability of utilities is necessary. In Section 4 a rationale is given why QALYs as VNM utilities can be considered to be fully interpersonally comparable.

\section{Aggregation of utilities}

Under classical utilitarianism social weifare was set equal to the sum of intuitively measurable and comparable individual utilities. These individual utili- 
ties were simply assumed to exist; no attention being paid to their origin. This concept of utility and social weifare was challenged by the Pareto school, which claimed that utility is an ordinal concept, reflecting only the indivicuai ordering of outcomes and being incomparable across individuals. Arrow's work on social choice lies within this Paretian tradition. In deriving his celebrated impossibility theorem ${ }^{4}$, Arrow defined a social welfare function (SWF) as a functional relation specifying a social ordering for any given $n$-tuple of individual orderings. By using only ordering information Arrow deliberately limited the informational framework, excluding all information on preference intensities. Arrow showed that if the number of individuals is finite and if the number of social states is greater than two, no SWF can satisfy the following four conditions: (i) unrestricted domain-the SWF should work for all logically possible individual orderings; (ii) weak Pareto-if every individual strictly prefers allocation $x$ to allocation $y$ then society should strictly prefer $x$ to $y$; (iii) non-dictatorship-there is no individual such that social preference is completely determined by the preferences of this individual regardless of the preferences of all other individuals in society; (iv) independence of irrelevant alternatives-social preference between two allocations should be independent of all other allocations. The requirement of independence of irrelevant alternatives excludes all information about other allocations and thereby inhibits the use of any information other than the individual orderings over $x$ and $y$. Using information on cardinal utility depends on the scaling of the utility function and this necessarily involves taking into account other atematives.

Various attempts have been undertaken to escape from Arrow's impossibility theorem by weakening his conditions. In this paper we consider the enrichment of the informational base of Arrow's social choice approach, i.e. a relaxation of Arrow's fourth condition, independence of irrelevant alternatives. A social welfare functional (SWFL) is defined as a rule that specifies exactly one social ordering for any given $n$-tuple of real-valued individual utility functions. Let $L_{i}$ be defined as the set of individual utility functions that are informationally equivalent, i.e. that provide the same information on individual preferences. For example, given Arrow's assumptions, all individual utility functions that are positive monotonic transformations are informationally equivalent. If individual utility is cardinally measurable, then elements of the set $L_{i}$ of informationally equivalent utility functions are positive linear transformations of each other: $U_{i}=a+b U_{i}^{\prime}, a \in \mathbb{R}$; $b>0 ; U_{i}, U_{i}^{\prime} \in L_{i}$.

A measurability set $L$ is defined as the set of all possible combinations of the

\footnotetext{
See Arrow (1950, 1951a). In these two works of Arrow the domain restriction was not defined tight enough as was pointed out by Blai (1957). The version in the second edition of the 1951 work (1963) is the best-known version.
} 
n-tuples of informationally equivalent individual utility functions. Depending on the assumptions about interpersonal comparability, the measurability set can be restricted. Combining the measurability assumptions about individual utilities with the assumptions about interpersonal comparability defines the measurability-comparability set $L *$. In Arrow's framework of social choice, where no interpersonal comparability is assumed and only the information revealed by individual orderings is incorporated. $L *$ consists of all individual utility functions that are positive monotonic transformations of each other. Sen $(1970,1977)$ distinguishes several other measurability-comparability combinations:

- cardinal non-comparability $-L *$ consists of all individual utility functions that are unique up to positive linear transformations;

- ordinal level comparability $-L *$ consists of all individual utility functions that are unique up to similar positive monotonic transformations;

- cardinal unit comparability $-L *$ consists of all individual utility functions that are unique up to location and common scale, i.e. $U_{i}=a_{i}+b U_{i}^{*}, a_{i} \in \mathbb{R}$ (the set of real numbers), $b>0$;

- cardinal full comparability $-L *$ consists of all individual utility functions that are unique up to common location and common scale, i.e. $U_{i}=a+b U_{i} *$, $a \in \mathbb{R}, b>0$.

Lemma $8 * 2$ in Sen (1970) shows that assuming cardinal non-comparability is not sufficient to solve Arrow's impossibility result. However, the other three informational frameworks are sufficient to remove the dilemma posed by Arrow's theorem. Clearly, it is interpersonal comparability that is crucial in enriching the informational basis of social cheice.

In cost utility analysis the calculation of the net advantage of one programme over another is of interest. For such an analysis to be relevant. units should be comparable. Location need not necessarily be common to all individuals, since in calculating net advantages the individual-specific locations are subtracted away and play no role in determining the relative effectiveness of programmes. Suppose for example that for a particular $n$-tuple of individual utility functions programme. $x$ is preferred to programme $y$. That is, $\Sigma\left[U_{i}\left(x_{i}\right)-U_{i}\left(y_{i}\right)\right]>0$, but also $\Sigma\left[a_{i}+\right.$ $\left.b U_{i}\left(x_{i}\right)-a_{i}+b U_{i}\left(y_{i}\right)\right]=b \Sigma\left[U_{i}\left(x_{i}\right)-U_{i}\left(y_{i}\right)\right]>0, a_{i} \in \mathbb{R}$ and $b>0$, and thus adding individual specific constants does not influence the relative effectiveness of programmes. If $b$ would be individual specific, which corresponds with cardinal non-comparability, $x$ might no longer be preferred to $y$. This suggests that in cost utility analyses we need only impose cardinal utility functions that have their scale in common. i.e. cardinal unit comparability. It seems not necessary to assume level comparability. However, several authors (e.g. d Aspremont and Gevers (1977); Sen (1977); Deschamps and Gevers (1978)) have shown that if cardinal unit comparability is assumed rather than cardinal full comparability, slightly strengthened versions of Arrow's conditions imply that the only possible SWFL is the utilitarian one. In such an informational framework, simply aggregating the number of utilities/QALYs over the relevant population is unobjectionable. This 
result was to be expected. The notion of equity involves special consideration being given to the badly off and this necessarily involves bringing in comparisons of utility levels. Given that the starting point of this paper was a concern for the equity consequences of utility-based decision making, a framework has to be imposed that allows such concerns to be justified. That is, a rationale must be given for assuming cardinal full comparability.

\section{4. von Neumann Morgenstern utilities}

If the vNM axioms hold then individual utility functions are cardinal, i.e. unique up to positive linear transformations. A possibility is therefore to use individual vNM utilities as an input in the social welfare functional. Taking individual vNM utilities as the basis from which social welfare judgements are to be derived was first proposed by Harsanyi (1955). Harsanyi's position has been severely criticized. The essence of the criticism was that vNM utilities are inextricably bound to situations involving risk. Arrow (1951a, p. 10): "...it (vNM utility theory) has nothing to do with welfare considerations, particularly if we are interested primarily in making a social choice among alternative policies in which no random elements enter. To say otherwise would be to assert that the distribution of the social income is to be governed by the tastes of individuals for gambling."

One can respond to such criticism in one of two ways. The first type of answer acknowledges that vNM utilities are only relevant in the context of risk, but asserts that health decision making typically involves risk and that, therefore, vNM utilities do have relevance in this context (e.g. Ben-Zion and Gafni (1983)). The second type of response challenges the assertion that vNM utilities only have relevance in the context of risk. According to this line of reasoning, cardinal utility has a meaning independent of risk. That cardinal utility has a meaning independent of risk has been criticized by Arrow (195lb) who writes about cardinal utility under certainty: "“...which is a meaningless concept anyway" (p. 425). Similar views have been expressed by Savage (1954), Ellsberg (1954), Luce and Raiffa (1957) and Fishburn (1989). Harsanyi (1987) on the other hand asserts that: "In fact, people's VNM utility functions are an important piece of information for welfare economics and ethics because they are natural measures for the intensity of people's desires, preferences and wants" (pp. 546-547). Wakker (1994) provides a defense for a unified notion of utility that does not need risk for its existence, but that has relevance for risk. Wakker observes that the development of expected utility theory by von Neumann and Morgenstern was motivated by their desire to obtain a cardinal utility that is relevant to game theory. The same cardinal utility, the expectation of which represents individual choices over lotteries over outcomes, is used as a unit of exchange between players in a game. Wakker (p. 8): 
"I think the applicability of risky utility as means of exchange between players is as questionabic as is apjlicability to welfare theory. or any other case of decisions under certainty." It cannot be excluded that VNM had in mind one notion of utility for the entire economic science. This viewpoint, that vNM utilities do indeed have relevance in other contexts than risk, underlies the discussion of QALYs as vNM utilities in this paper.

Having provided a rationale for using cardinal (vNM) utilities as the foundation of social welfare judgements, the question remains how interpersonal comparability can be ensured given that individual utilities are unique only up to positive linear transformations and given that scaling up the utility of one individual, while keeping the utilities of the other individuals constant, may aiter the outcome of the social choice problem. Hildreth (1953) suggested considering two specialiy defined outcomes $X$ and $Y$, such that everyone prefers $X$ to $Y$, and to assign predefined real values to these social states. This makes individual utility functions interpersonally comparable. In fact this approach is typically used in cost utility analysis. The general approach to aggregation. as outlined for example by Williams (1981) and Torrance (1986), is to assign a utility of zero to death and a utility of one to normal or full health and to regard a year of healthy life as being of equal intrinsic value to everyone. Torrance and Feeny (1989) have argued that it may be more in line with existing practice to assume that a life in full health has equal value for everyone. Applying this scaling, moreover, avoids the problem that utilities have to be assigned to health states without specifying the time dimension of the health states. Gafni and Torrance (1984) have argued that health states have a time dimension inextricably bound to them and that it is impossible to measure utilities for health without specifying a time dimension. Gafni and Birch (1991) have argued that the common way to measure $\mathrm{vNM}$ utilities is inconsistent with the criterion that a life in full health is of equal value to everyone. They have provided an algorithm to measure utilities that are consistent with this criterion. In their approach the vNM utility function is scaled such that a life in full health receives utility one and immediate death utility zero. This approach guarantees that individual utilities are consistently scaled and interpersonally comparable. A disadvantage of the method proposed by Gafni and Birch is that two rather that one standard gamble questions are necessary to determine health state utilities. The measurement task therefore becomes more involved.

Summarizing. the above discussion establishes a rationale for aggregating QALYs as (vNM) utilities. Section 3 showed the need for cardinal fully comparable utilities if we are to allow distributional considerations to play a role in cost utility analysis. vNM utility theory establishes cardinality of the individual utilities. Following Wakker's argument a case can be made for the assertion that VNM utilities do indeed have relevance in the context of welfare judgments. Finally, by Hildreth's approach, which is typically followed in cost utility analysis, a consistent scaling procedure emerges, which ensures that individual vNM utilities can be interpersonally compared in a meaningful way. 


\section{QALY utilitarianism}

\subsection{Notation and structural assumptions}

This subsection introduces notation and structural assumptions. Denote the set of QALY allocations by $X$. A typical element of the set $X$ is a vector $\mathbf{x}=\left(x_{1}, \ldots\right.$, $x_{n}$ ) representing an allocation of QALYs resulting from the implementation of a health care programme with each $x_{i}$ indicating the number of QALYs received by individual $i$ and $n$ being the number of individuals affected by the programme. Assume without loss of generality that for each individual the possible number of QALYs is non-negative, i.e. $X \in \mathbb{R}_{+}$. We are interested in the social preference relation over the set of QALY allocations, meaning "at least as good as". Let $\succ$ and $\sim$ denote its asymmetric and symmetric part, respectively. Throughout $\geq$ is assumed to be a weak order. That is, $\geq$ is complete, either $x \geq y$ or $y \geq x$ or both, and transitive, if $x \geq y$ and $y \geq z$ then $x \geq z$. Moreover, $\geq$ is assumed to be continuous. Continuity of the preference relation guarantees that if a real-valued function is defined over $X$, this function has an interval as its image.

Denote by $x_{-i} v_{i}$ the vector $\mathbf{x}$ with coordinate $i$ (the number of QALYs individual $i$ receives) replaced by $v_{i}: x_{-i} v_{i}=\left(x_{1}, x_{2}, \ldots, x_{i-1}, v_{i}, x_{i+1}, \ldots\right.$, $x_{n}$ ). Let $A$ be a subset of the individuals affected by a health care programme: $A \subset I=\{1,2, \ldots, n\}$. Then $x_{-} A_{A}$ denotes the vector $\mathrm{x}$ in which for all individuals in subset $A x_{i}$ is replaced by $v_{i}$. For example if $A=\{1,2,3\}$, then $x_{-A} v_{A}=\left(v_{1}, v_{2}, v_{3}, x_{4}, \ldots, x_{n}\right)$. Denote by $z_{i}$ the individual preference relation "at least as good as". As before, $>_{i}$ and $\sim_{i}$ are defined as the asymmetric and symmetric part of $\geq_{i}$, respectively.

Let $Z$ be a set of probability distributions over the set of QALY allocations $X$. A typical element of $Z$ is $\left(p^{1}, x^{1}, \ldots, p^{m}, x^{m}\right)$ where allocation $x^{j}$ occurs with probability $p^{j}$ and $m$ can be any natural number. Let $\gtrsim$, be a social preference relation defined on $Z$. Throughout the paper it is assumed that individual preference relations over probability distributions satisfy the von Neumann Morgenstern (vNM) axioms. In the formulation by Jensen (1967), a preference relation $\geq$ ' satisfies the vNM axioms if: (i) $Z^{\prime}$ is a weak order; (ii) vNM independence, $P \geq Q \Leftrightarrow(\mu P+(1-\mu) R) \geq '(\mu Q+(1-\mu) R) \forall 0<\mu<1$ and $P, Q, R \in Z$; (iii) Jensen continuity, $\left(P \succ^{\prime} Q, Q \succ^{\prime} R\right) \Rightarrow \kappa P+(1-\kappa) R \succ^{\prime} Q$ and $Q \succ^{\prime} \rho P+(1$ $-\rho) R$ for some $\kappa, \rho \in(0,1)$. If a preference relation satisfies the vNM axioms, a cardinal real-valued utility function exists, the expected value of which represents the preference relation.

\subsection{Derication of $Q A L Y$ utilitarianism}

We will derive (QALY) utilitarianism by adding a condition to the axiomatic framework of Harsanyi (1955) in which a partial characterization of utilitarianism is given. The method of proof differs from the proof given by Harsanyi in that use 
is made of a result developed by Fishburn (1965). Furthermore, by using a theorem from Maas and Wakker (1994) the utility function is shown to be continuous. Continuity is important to establish. If QALYs (real numbers) are added up across individuals the social utility function is implicitly assumed to be continuous. However Harsanyi's result does not imply this.

Marsanyi noi uniy icyuited individual greferences to satisfy the vNM axioms, as has been assumed in Section 5.1, but also required social preferences to satisfy the vNM axioms. According to Harsanyi the vNM axioms are essential requirements of rationality, much in the same spirit as Arrow considered weak ordering to be a basic requirement of rationality of social preferences. Furthermore, Harsanyi imposed the following condition:

Condition $\boldsymbol{H}$-if two alternatives, defined by probability distributions over the set of outcomes, are indifferent from the standpoint of every individual, then they are also indifferent from a social standpoint.

As shown by Harsanyi (theorem V), these three conditions allow the derivation of the SWFL as a weighted sum of the individual utilities:

$$
U(x)=\sum_{i=1}^{n} A_{i} U_{i}\left(x_{i}\right)
$$

This is not a full characterization of QALY-utilitarianism, given that the scaling factors $\lambda_{i}$ may differ between individuals and the utility functions are individualspecific. QALYs are assumed to be similar across individuals. Therefore a condition has to be added to ensure this similarity.

A permutation $\boldsymbol{\pi}$ of the $\boldsymbol{n}$ individuals is a function specifying a rearrangenent of the individuals. Denote by $\pi(i)$ the permuted value of $i$. Now consider the following condition:

Condition $A$ (anonymity) $-\left(U_{i}\right) \sim\left(U_{z(i)}\right)$ for all $\left(U_{i}\right)=\left(U_{i} \ldots \ldots U_{n}\right)$ and permutation functions $\pi$ on $I=\{1, \ldots, n\}$.

Condition $A$ asserts that social indifference should hold between a utility/QALY allocation $\boldsymbol{x}$ and any utility/QALY allocation $\boldsymbol{y}$ which is a permutation of $\boldsymbol{x}$. Condition $A$ ensures that social preference is independent of who gets which utility/QALY. For example, in the hypothetical situation that there is one additional QALY to be divided between two individuals with a similar endowment of QALYs ${ }^{5}$. by condition $A$, society should have no preference as to which individual will receive this additional QALY. However, condition $A$ is weaker than what is referred to in the cost utility literature as "a QALY is a QALY no matter who gets it". According to the latter, society should in every situation be indifferent with respect to who gets a QALY. Condition $A$ only says that in case

\footnotetext{
"Note that the impast of other endowments, e.g. income, is ignored by condition $A$. These could be incorporated by making health utilities dependent of these endowments. For example, if health state uilitics are income dependent, condition $A$ has implications for income dependent health utilities.
} 
one QALY allocation is a permutation of another, indifference shouid hold. For example, suppose a programme has resulted in a QALY allocation $(3,1)$, i.e. individual $a$ has received three QALYs and individual $b$ has received one QALY, and one more QALY is to be allocated. Then by the argument that "a QALY is a QALY no matter who gets it" society should be indifferent between allocations $(4,1)$ and $(3,2)$. However, condition $A$ does not provide guidance with respect to social preference between $(4, i)$ and $(3,2)$. Condition $A$ asserts that if society prefers $(3,2)$ to $(4,1)$ then it should also prefer $(2,3)$ to $(1,4)$ when the initial allocation is $(1,3)$ : by condition $A(2,3) \sim(3,2)$, we know that $(3,2) \succ(4,1)$, applying condition $A$ once again gives $(4,1) \sim(1,4)$ and thus by transitivity $(2$, $3)>(1,4)$. Imposing condition $A$ on top of Harsanyi's conditions is necessary and sufficient for QALY uilitarianism.

Theorem 1-the following two statements are equivalent:

(i) The social preference relation $\geq$ : can be represented by QALY utilitarianism:

$$
U(x)=\sum_{i=1}^{n} U\left(x_{i}\right)
$$

(ii) Both individual and social preferences satisfy the vNM axioms and moreover conditions $H$ and $A$ hold. Furthermore, $U$ is continuous and unique up to positive linear transformations.

A proof of this recult can be found in Appendix A.

\section{Ex ante versus ex post equity}

Theorem 1 has been derived by imposing four conditions: that individual preferences satisfy the vNM axioms. that social preferences satisfy the vNM axioms, condition $H$ and condition $A$. In the remainder of the paper we continue to require that individual preferences satisfy the $\mathrm{vNM}$ axioms. Particularly during the last two decades much empirical evidence has been presented that descriptively individual preferences frequently violate these axioms. Normatively the axioms still have considerable force and are appealing enough to adhere to. We will also continue to assume that condition $A$ holds. Condition $A$ asserts that the identity of a QALY recipient should play no role in health decision making. Condition $A$ ensures that the principle that a life in full health should be equal for all individuals holds, and thereby allows consistent scaling of the utility functions according to the equity principles developed by Gafni and Birch (1991). Moreover, as the example in the previous section shows, condition $A$ does not predict choice with respect to every allocation and therefore allows additional equity principles to be imposed. 
The remainder of the paper examines the consequences of relaxing the two remaining conditions, that social preferences satisfy the $\mathrm{vNM}$ axioms and condition $\boldsymbol{H}$. The restrictiveness of these assumptions can be illustrated by means of an example. Consider two individuals (or equivalently two groups of individuals) and two possible ssates of the world. $X$ and $Y$, each with a probability of occurrence of 0.5. This gronahility is knewn is iviti inciviciuats. Consider the following tivee health care programmes each resulting in different QALY allocations:

$\begin{array}{llll}\text { Programme } & \text { State } X & \text { State } Y & \text { Expected utility } \\ 1 & (1,0) & (1,0) & 1 \\ 2 & (1,0) & (0,1) & 1 \\ 3 & (1,1) & (0,0) & 1\end{array}$

Under the assumptions being made, by Theorem 1, social indifference should hold between the three health care programmes, given that the expected utilities of the three programmes are equal. However, it is conceivable that the decision maker will prefer programmes 2 and 3 to programme 1 given that the former two programmes offer both individuals a possibility of receiving a QALY, whereas programme 1 denies the second individual the possibility of receiving a QALY. Diamond $(1967)^{6}$ has argued that it is essentially vNM independence that requires indifference to hold in the above example. (See also Sen (1976), Broome (1982) and Ulph (1982). For a counter-argument see Harsanyi (1975).) This is most easily seen by comparing programmes 1 and 2 . Under condition $A$, the decision maker is indifferent between the outcomes of the two programmis when state $Y$ occurs. However, under state $X$ the outcomes of the two programmes are equal and therefore, by vNM independence, overall indifference should prevail. On the other hand, if the decision maker is concerned with the fairness of the allocation process, generally referred to as ex ante equity, programme 2 should be chosen, because this offers both individuals a possibility of obtaining a QALY. Incorporating ex ante equity concerns implies dismissing the requirement that social preferences satisfy the VNM axioms. Incorporating ex ante equity considerations can be ensured by imposing the following ex ante equity condition on the social preference relation:

Condition $\mathrm{E}$-if $p_{k}=q_{k}$ for all $k \in I \backslash\{i, j\} ; p_{i}+p_{j}=q_{i}+q_{j}$ and $\left|p_{i}-p_{j}\right|<$ $\left|q_{i}-q_{j}\right|$ then $P>Z Q$. $^{7}$

where $P$ and $Q$ are lotteries over $X$ and the $p_{i} s$ and $q_{i}$ s are marginal probabilities, indicating the probability that individual $i$ receives a given amount of QALYs, $Q^{c}$. In words condition $E$ states the following. Suppose all individuals, other than $i$

\footnotetext{
${ }^{6}$ See also Sen (1976). Broone (1982) and Ulph (1982). For a counterargument see Harsanyi (1975).

${ }^{7}$ Condition $E$ is comparable to Fishburn's axiom of risk-sharing equity in the context of public risk evaluation (Fishburn. 1984). See also Fishburn and Straffin (1989).
} 
and $j$, have the same marginal probability of receiving $Q^{c}$ under two health care programmes (in the above example $Q^{c}$ is equal to one). Taken together $i$ and $j$ have the same marginal probability of receiving $Q^{c}$, but in one programme this marginal probability is more equally divided between the two individuals (in the situationi distribution $P$ ). Then, by condition $E$, the programme with the more equal distribution of marginal probabilities over $i$ and $j$ is to be preferred to the one that leads to a less equal distribution of marginal probabilities over $i$ and $j$.

Condition $E$ is not incompatible with condition $\boldsymbol{H}$. Condition $E$ dictates how differences in marginal probabilities should affect social preference, whereas condition $\boldsymbol{H}$ dictates how equality of marginal probabilities should affect social preference.

It is also conceivable that, in choosing between programmes 2 and 3 , a social decision maker prefers programme 3 to programme 2, given that programme 3 guarantees an equal distribution of QALYs under both states of the world, whereas programme 2 necessarily leads to a situation of inequality. This preference is determined by a concern for the final distribution of QALYs, often referred to as ex post equity. Let $p_{i-j}$ denote the probability of the event that only individual $i$ gets a QALY and let $p_{i+}$, denote the probability of the event that both individual $i$ and individual $j$ receive a QALY. In the exampie above, $p_{i-j}$ is 0.5 for programme 2 and 0 for programme 3, whereas $p_{i+j}$ is 0 for programme 2 and 0.5 for programme 3. Incorporating a concern for ex post equity can be establiched by imposing tixe foliowing condition on the social preference relation:

Condition $P$-if $P=Q, P, Q \in Z$ apart from $p_{i-j}=q_{i-j}-\gamma, p_{j-i}=q_{j-i}-\gamma$, $p_{i+j}=q_{i+j}+\gamma$. with $\gamma>0$, then $P \succ_{i} Q .{ }^{8}$

It can easily be seen that condition $P$ is incompatible with condition $H$. In the situation described by condition $P, p_{i}=q_{i}$ for all individuals, so by condition $H$, $P \sim Q$. However, probability distribution $P$ offers a greater probability of individuals $i$ and $j$ both receiving a QALX. Therefore, by condition $P, P \succ_{Z} Q$. Thus, incorporating ex post equity considerations in health care decision making means rejection of condition $\boldsymbol{H}$. Condition $\boldsymbol{H}$, innocuous as it may appear, has the effect of making social choice dependent on individual preferences only. The condition leaves no room for supra-individual interests. Incorporating distributional concerns therefore means relaxing the condition that social choice depends only on individual preferences and allowing complementarity between individual utility/health levels. In the next section two approaches are discussed to incorporate such complementarity.

\footnotetext{
${ }^{8}$ This condition is the converse of Keeney"s assumption of catastrophe avoidance (Keeney, 1980). Fishburn (1984) and Fishbum and Straffin (1989) have developed similar "common fate equity" axioms for the context of public risk evaluation.
} 


\section{Ex post equity algorithms for QALY aggregation}

\subsection{A multiplicatice social utility function}

As has been observed in the proof of Theorem 1. condition $H$ is equivalent to additive independence (Fistburn, 1965, 1970). Therefore, a way to introduce complementarity is to translate generalizations of additive independence. known from the literature on multi-attribute utility theory, to the context of social choice. One possibility is to impose the analogue of mutual utility independence on the social preference relation. ${ }^{9}$ Mutual utility independence is a preference coridition that is entirely formulated in terms of lotteries on outcomes. I deviate slightly from this approach by making as little use of lotteries as possible in the conditions imposed on the social preference relation. The main motivation underlying my approach is that lotteries are highly artificial constructs that are typically not available in real-world health decision situations. This does not imply that lotteries have no role to play in health decision making. They are, for example, necessary in standard gamble measurements. The reason I have avoided the use of conditions that are formulated in terms of lotteries is that in my opinion such conditions are harder to understand than conditions that are formulated in terms of certainty. The ultimate aim of characterizations is to clarify what assumptions a particular representation depends on. The easier the preference conditions are to understand the easier it is io assess the appeal of representations. An additional motivation to use the independence condition $S E$ stated below is that this condition is more common in social choice theory and that a justification for imposing it has been given. ${ }^{\text {to }}$

Condition $S E$ - the social preference relatiun satisfies condition $S E$ if for all QALY allocations $x, x^{\prime}, y, y^{\prime} \in X$, for all subsets of individuals $A \subset I=\{1, \ldots \ldots$ n):

$$
\left[v_{-A} x_{A} \geq v_{-A} y_{A}\right] \Leftrightarrow\left[w_{-A} x_{A} \geq w_{-A} y_{A}\right]
$$

By condition $S E$. individuals who are indifferent between two QALY allocations. the individuats who are not in subset $A$, exert no influence on social preference. Condition $S E$ is the analogue of the "sure thing principle" in decision making under uncertainty (Savage, 1954) and of "complete strict separability" in consumer theory (Blackorby et al., 1978). Condition SE underlies the current practice of using incremental analysis in cost utility analysis (see. for example. Drummond et al., 1987). Incremental analysis prescribes how to calculate the net advantage of one programme over another. The implicition of this is that if two programmes produce the same amount of QALYS for certain individuals, then

\footnotetext{
"For a detinition of mutual utility independence see, for example. Keeney and Raiffa (1976. p. 289).

${ }^{10}$ For a defense see Fleming (1952); Deschamps and Gevers (1978): Sen (1976. 1977).
} 
these individuals do not influence the outcome of the analysis. This is exactly what condition $S E$ asserts.

Condition $S E$ is entirely formulated under conditions of certainty. However, because we seek a representation for the social preference relation under conditions of risk, we have to impose a condition which is defined with respect to preferences under risk. Consider the following condition:

Condition $U I$-let $B$ be a subset of individuals, i.e. $B \subset I=\{1, \ldots, n\}$, let y be a particular constant QALY allocation, $y \in X$, and let $\geq: \mid y$ be the preference relation defined over probability distributions on $\mathbb{R}_{+}^{B}$ by fixing the values of those individuals outside subset $B(I-B)$ at levels identical to those of $y . B$ is utility independent if $\geq .1 \mathrm{v}$ is independent of the constant value at which $\mathbf{y}$ is fixed.

In the special case where all probability distributions are degenerate, i.e. one outcome results with probability one, condition $U I$ is equivalent to condition $S E$. If condition $U I$ holds for all subsets of individuals $B$, mutual utility independence holds. However, in combination with condition $S E$ it is not necessary to impose condition $U I$ for all subsets of individuals. It is sufficient to impose that $U I$ holds for one individual. Thus, if all other $n-1$ individuals are indifferent between two QALY allocations, then social preferences for lotteries on these two allocations are governed by the preferences of this particular individual. Denote this condition as $U I^{\prime}$. Condition $U I^{\prime}$ only holds when all other individuals are indifferent. The relevant individual can therefore not be considered to be a dictator in Arrow's sense. Condition $U I^{1}$ is an artificial condition, because situations like the one described in the condition will rarely occur and this makes it hard to assess the condition. However, condition $U I^{1}$ is not very restrictive in terms of the social preference relation. If the one individual for who condition $U I^{1}$ holds, is the individual who is worst off in terms of health. then it seems defensible to impose that. in case all other individuals are indifferent, social preferences under risk should be governed by the preferences under risk of this individual.

A second theorem can now be given.

Theorem 2-the following two statements are equivalent:

(i) The social preference relation can be represented by

$$
U(x)=(1 / \lambda) \prod_{i=1}^{n}\left[\lambda U\left(x_{i}\right)+1\right]-(1 / \lambda)
$$

where $U(x)$ is a continuous social utility function, unique up to positive linear transformations and scaled between 0 and 1 , the $U\left(x_{i}\right)$ are identical additive utility functions, that can be interpreted as (rescaled) QALYs and $\lambda$ is a scaling constant, that is not equal to zero.

(ii) Both individual and social preferences satisfy the vNM axioms; social preferences satisfy conditions $A, S E$ and $U I^{\prime}$.

If condition $P$ also holds then $\lambda>0$.

A proof of this result can be found in Appendix A. 


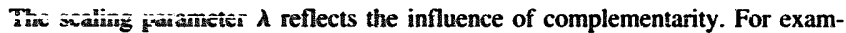
ple, for two individuals Eq. (4) reduces to

$$
U(x)=U\left(x_{1}\right)+U\left(x_{2}\right)+\lambda U\left(x_{1}\right) U\left(x_{2}\right)
$$

If $\lambda>0$, complementarity increases social utility, which is the effect of imposing suisulition $P$.

Consider the example of the two individuals " and the three health care programmes descri hed in Section 6. Recalculating the social utility of health care programmes 1,2 and 3 gives 1,1 and $1+0.5 \lambda$, respectively. Thus, under condition $P$, programme 3 is now preferred, which is consistent with the (imposed) preference for ex post equity. Indifference still holds between programmes 1 and 2 , because they have the same distributional implications. Indifference between programmes 1 and 2 reflects the fact that ex ante equity has not been taken into account.

The value of $\lambda$ reflect: views on equity. These views can either be those of a policy maker or those of the general public. The question whose views are more appropriate is not the subject matter of this paper. Views on equity can partly be expressed by conditions such as condition $\boldsymbol{P}$, but to cetermine the relative weight given to aggregating the individual QALYs (the efficiency side) and to complementarity between individual QALYs (the ex post equity side) requires explicit choices with respect to the equity-efficiency trade-off. Trading off attributes is common practice in multi-attribute utility theory and the tools of multi-attribute utility theory can be of great help in eliciting preferences between efficiency and equity in health care.

Under condition $A$ rne trade-off question is sufficient to determine $\lambda$. As an illustration, cousider again the example of two individuals. In Theorem 2. the $U\left(x_{i}\right)$ are rescaled QALYs (for more details see the proof of Theorem 2 in Appendix A): $A_{i} U^{\prime}\left(x_{i}\right)$ where all $\lambda_{i}$ are equal and positive and $U^{\prime}\left(x_{i}\right)$ indicates the number of QALYs sach individual receives. For the purpose of the theorem this was no problem given that VNM utility functions are unique up to positive linear transformations. However, to calculate $\lambda$ we need to determine $\lambda_{i}$. This can be done by asking for tha: indifference probability $p$ in the choice between $(1,0)^{12}$ with certainty and a ganble with outcomes (1. 1) with probability $p$ and $(0,0)$ with probability $(1-p)$. Suppose the indifference probability is 0.4 . Scale $U(x)$ such that $U(1,1)=1$. Then, substituting values in Eq. (5), $1=0.4 * 1+0.4 * 1+$ $\lambda * 0.4 * 1 * 0.4 * 1$. This gives $\lambda=1.25$. It is conceivable that exact values for $\lambda$ cannot be specified ir every situation. but only a range of values. In that case it seems sensible to include this range of values for $\lambda$ in sensitivity analyses.

\footnotetext{
"To be formally correct, for two individuals a stronger condition than $S E$ has to be imposed: the analoge of Wakker's hexagun conditicn w'sich will be discussed in Section 8 (Wakker, 1989).

is $\mathrm{Or}(0.1)$ which. under condition $A$. is equav dient to $(\mathrm{i}, 0)$.
} 
Finally, the multiplicative social utility function, as derived above, only incorporates equity concerns to a limited extent. By condition $S E$, indifferent individuals do not exert an influence on social preference. In a situation where the non-indifferent individuals are already in a good health state but the indifferent individuals are in an appalling health state, it may be preferred that the non-indifferent individuals do not receive more QALYs in order to prevent a more unequal distribution of health (utility). Such equity concerns cannot be accommodated by the proposed multiplicative social utility function. In the next section we propose a social utility function that is able to embrace ex post equity concerns in a more comprehensive way.

\subsection{A two-component social utility function}

Continue to assume that social preferences satisfy the vNM axioms. Therefore, as in Sections 5 and 7.1, the social preference relation is defined over probability distributions. We propose a method that allows the decision maker in simultaneously consider the maximization of QALYs, that can both be interpreted as health and utility, and the distribution of these QALYs, that is ex post equity. The idea is to assess a two-component social utility function $U(y)=U\left(y_{1}, y_{2}\right)$, the components of which are the total number of QALYs $\left(y_{1}\right)$ and a real valued summary index reflecting the ex post distribution of these QALYs $\left(y_{2}\right)$. The set of outcomes, $Y$, is assumed to satisfy certain structural assumptions. ${ }^{13}$ The assessment of such a two-component multi-attribute utility function becomes much easier if the following assumption can be accepted.

Condition $\mathrm{TCl}$ (two-component independence)-if two lotteries induce the same probability distribution over $Y_{1}$ (total number of QALYs gained) and the same probability distribution over $Y_{2}$ (the summary indix reflecting the ex post distribution), then these lotteries are indifferent.

This condition is sinilar to additive independence (and to condition $H$ for the special case of two individuals) and guarantees, in combination with the assumption that the social preference relation satisfies the vNM axioms, by Theorem 2 in Fishburn (1965) that $U(y)$ is additive:

$$
U(y)=\lambda_{1} U_{1}\left(y_{1}\right)+\lambda_{2} U_{2}\left(y_{2}\right)
$$

where $U, U_{1}$ and $U_{2}$ are scaled $\mathrm{vNM}$ utility functions, and $\lambda_{1}$ and $\lambda_{2}$ are scaling constants that reflect views on the trade-off between "efficiency" in the sense of the maximization of QALYs and ex post equity.

\footnotetext{
${ }^{13} Y$ is assumed to the a Cartesian product of $Y_{1}$ and $Y_{2}, Y_{1} \in I R_{+} \backslash\{0\}$ and $Y_{2} \in I R_{+}$. The reason that 0 is excluded from $Y_{1}$ is that otherwise not every value from $Y_{1}$ can be comonned with every value from $Y_{2}$ and. by consequence, $Y$ cannot be a Cartesian product.
} 
The summary index defined over the ex post distribution should satisfy certain properties. For example, it should be sensitive to a transfer from an individual who is relatively well off in terms of the number of QALYs received from the implementation of a health care programme, to an individual who receives less QALYs from this programme. An example of such a summary index is Theil's entropy measure (cf. Sen. 1973):

$$
y_{2}=\sum_{i=1}^{n} x_{i} \ln \left(n x_{i}\right)
$$

where $x_{i}$ denotes the share of the total amount of QALYs received by individual i. $y_{2}$ increases with inequality in the QALY distribution, therefore, under condition $P, \lambda_{2} U\left(y_{2}\right)$ must have a negative sign.

Assume that condition $\mathrm{TCl}$ holds. Assume further that the utility function for the amount of QALYs is linear ${ }^{14}$ and that the utility function for the ex post distribution is equal to Theil's entropy index. Then for the example in Section 6 we obtain: $U$ (programme 1$)=U($ programme 2$)=\lambda_{1}+\lambda_{2}$ ln 2; $U$ (programme $3)=\lambda_{1}$. Under condition $P \lambda_{2}<0$, and thus programme 3 is preferred, consistent with a preference for ex post equity.

Only one trade-off question has to be asked to determine the scaling constants, $\lambda_{1}$ and $\lambda_{2}$. Suppose that a programme yields benefits for two groups of individuals and that the maximum amount of QALYs the programme can generate is 100 . Then the best possible outcome is $(50,50)$ : the number of QALYs is maximized and there is no inequality. Scale $U($.$) such that U(50,50)=1$. The worst outcome is $(x, 0)$ in which $x$ is infinitesimally small: the number of QALYs is minimized and there is complete inequality. Let $U(x, 0)$ be zero. Now $\lambda_{1}$ can be determined by eliciting the indifference probability in a choice between $(100,0)$, i.e. the number of QALY's is at its maximum, but inequality is complete, for certain and a gamble giving $(50.50)$ with probability $p$ and $(x, 0)$ with probability $(1-p)$. Suppose $p=0.85$. Substituting in Eq. (6) gives: $U(100,0)=\lambda_{1} * 1=p$. Thus $\lambda_{1}=0.85$ and $\lambda_{2}$ is by consequence equal to 0.15 .

If condition $\mathrm{TCl}$ does not hold, complementarity between $y_{1}$ and $y_{2}$ has to be introduced in the model. For example. if the social preference relation does not satisfy condition $\mathrm{TCl}$, but does satisfy a somewhat stronger condition than $J E$ (the hexagon condition), and does satisfy $U I^{\prime}$, then the term $\lambda \lambda_{1} \lambda_{2} U_{1}\left(y_{1}\right) U_{2}\left(y_{2}\right)$ should be added to the additive form, reflecting complementarity. In this case one additional trade-off question has to be asked to determine the scaling constants.

\footnotetext{
This assumption may appeas restrictive. However, it reflects the impact of the efficiency sice in the decisicia makisis process: one of the arguments of the overall utility function is the maximization of the amouns of QALYs. To represent the efficiency side of the decision making process the total anount of QALYs seems a good indicator. If the linear function is not believed to be appropriate, alternative functional forms can simply be subitituted.
} 


\section{Algorithms incorporating both ex post and ex ante equity}

In Section 6 it was argued that if a concern for ex ante equity is to be incorporated in social preference, the vNM utility function can no longer be used. Therefore, in this section, rather than taking a preference relation over probability distributions as primitive, a representation is sought for a preference relation under certainty. In this section we consider a three component social value function $V(y)=V\left(y_{1}, y_{2}, y_{3}\right)$, where $y_{1}, y_{2}$ and $y_{3}$ denote the number of QALYs gained, which can be both utilities and health, and real valued summary indices reflecting the ex post equity and the ex ante equity of the QALY allocation process, respectively. More specifically, we will derive a representation for the value function $V\left(y_{1}^{c e}, y_{3}\right)^{15}$ in which $y_{i}^{c e}$ denotes the certainty equivalent amount of QALYs gained, with the ex post equity index held fixed, for probability distributions over $y_{1}$ and $y_{2}$. For example, if the ex post equity index is fixed at its optimal value corresponding with no inequality, then for every lottery the equivalent number of equally distributed QALYs is determined. Under the assumption that social preferences increase monotonically with the number of QALYs, which seems reasonable and is typically assumed in cost utility analysis, the equivalent number of QALYs will consistently rank order lotteries, a higher number corresponding to more preferred. $V\left(y_{1}^{c e}, y_{3}\right)$ is equivalent to $V\left(U\left(y_{1}, y_{2}\right), y_{3}\right)$ in which $U$ is a vNM utility function defined over $y_{1}$ and $y_{2}$. By means of the so $:$ dal value function the certainty equivalent number of QALYs of a gamble can be traded off against its ex ante equity implications. Thus we continue to assume that social preferences with respect to lotteries over $y_{1}$ and $y_{2}$ while holding $y_{3}$ fixed satisfy the vNM axioms. vNM utility functions are still used to evaluate attributes $y_{1}$ and $y_{2}$. because ex ante equity is defined in the context of risk and therefore utility functions that are applicable in the context of decision making under risk are called for. Social policy making is essentially a normative decision problem, and to date there is no theory that challenges expected utility theory as a normative theory of decision making under risk. Diamond's objection against vNM utility theory concerned its implications for ex ante equity (Diamond, 1967). His argument does not conflict with the use of vNM utility functions to evaluate $y_{1}$ and $y_{2}$. We will describe the preference conditions that make it possible to represent $V(y)$ by the following simple expression:

$$
V(y)=\kappa_{1} V_{1}\left[\lambda_{1} U_{1}\left(y_{1}\right)+\lambda_{2} U_{2}\left(y_{2}\right)\right]+\kappa_{3} V_{3}\left(y_{3}\right)
$$

where $U_{1}$ and $U_{2}$ are (scaled) vNM utiity functions, and $V_{1}$ and $V_{3}$ are (scaled) value functions.

\footnotetext{
${ }^{15}$ The set $Y$ is again assumed to be a Cartesian product set. It is assumed that $Y_{1} \in I R_{+} \backslash(0): Y_{2}$. $Y_{3} \in R_{+}$. In combination with the assumption that $\geq$on $Y$ is a weak order. this guarantees the existence of $V(Y)$. If $\geq$ on $Y$ is morever assumed to be continuous, then $V(Y)$ will be continuous.
} 
Consider the following preference condition:

Hexagon condition-if $\left[\left(y_{1}^{c e t}, y_{3}\right) \sim\left(y_{1}^{c e}, y_{3}^{\prime}\right)\right.$ and $\left(y_{1}^{c e n}, y_{3}\right) \sim\left(y_{1}^{c e t}, y_{3}^{\prime}\right)$ and $\left.\left(y_{1}^{c e t}, y_{3}^{\prime}\right) \sim\left(y_{1}^{c e}, y_{3}^{\prime \prime}\right)\right]$ then $\left(y_{1}^{c e t}, y_{3}^{\prime}\right) \sim\left(y_{1}^{c e}, y_{3}^{\prime \prime}\right)$.

Suppose that $y_{1}^{\text {cet }}>y_{1}^{\text {cet }}>y_{1}^{e c}$ and that $y_{3}^{\prime \prime}>y_{3}^{\prime}>y_{3}$. By the first two indifferences in the hexagon condition, both the utility difference between $y_{1}^{\text {ces }}$ and $y_{1}^{\text {ce }}$ and the utility difference between $y_{i}^{\text {cet }}$ and $y_{1}^{\text {ct }}$ are just sufficient to compensate the utility difference between $y_{3}^{\prime}$ and $y_{3}$. Then the third and the (implied) fourth indifierence assert that if the utility difference between $y_{1}^{c e s}$ and $y_{1}^{c e}$ is also just sufficient to compensate the utility difference between $y_{3}^{\prime \prime}$ and $y_{3}^{\prime}$, then the utility difference between $y_{1}^{\text {cet }}$ and $y_{1}^{\text {cer }}$ should also be just sufficient to compensate the utility difference between $y_{3}^{\prime \prime}$ and $y_{3}^{\prime}$. The hexagon condition is, under transitivity of the indifference relation, implied by the Thomsen condition, which has been more commonly used as a characterizing condition for an additive two attribute utility function (e.g. Debreu, 1960).

Given that the hexagon condition holds, a preference relation $\geq_{A}$ can be defined over probsability distributions on $y_{1}$ and $y_{2}$, while fixing $y_{3}$ at some constant reference value. This preference relation is assumed to satisfy the vNM axiurss and condition $\mathrm{TCl}$ (two-component independence).

The following rsult can then be sîted.

Theorem 3-the following are equivalent:

(i) $V(Y)$ can be represented by Eq. (8).

(ii) The social preference relation on $Y$ is a continuous weak order that satisfies

the hexagon condition. and $\geq_{A}$ satisfies the vNM axioms and condition $T C I$.

Furthermore. $V, U_{1}, U_{2}$ and $V_{3}$ are continuous and unique up to positive linear transformations. The $\lambda_{i}$ s are scaling constants.

A proof of this theorein can be found in Appendix A.

The summary index $y_{3}$, reflecting ex ante equity, should be sensitive to changes between individuals in the marginal probability of obtaining a given amount of QALYs, $Q^{r}$. The following index has this property:

$$
y_{3}=(1 / n) \sum_{i=1}^{n}\left(q_{i}-q_{m}\right)^{2}
$$

where $q_{i}$ denotes the marginal probability of individual $i$ receiving $Q^{c}$ and $q_{m}$ denotes the mean probability of receiving $Q^{c}$. A more equal distribution of marginal probabilities leads to a lower value for the summary index. Therefore, under condition $E, \kappa_{3} V_{3}\left(y_{3}\right)$ should be negative. The amount of QALYs with respect to which $q_{i}$ and $q_{m}$ are defined should be chosen according to what it is believed that individuals are entitled to. For example, if the conviction exists that every individual should have an equal probability of receiving a life in full health then $q_{i}$ denotes the individual probability of obtaining a life in full health.

Suppose with respect to the example of Section 6 that the conditions of Theorem 3 hold and that $U_{1}, U_{2}, V_{1}$ and $V_{3}$ are identity functions, i.e. $U_{i}\left(y_{i}\right)=y_{i}$, with $y_{2}$ and $y_{3}$ as in Eqs. (7) and (9), respectively. Then $V($ programme $i)=\kappa_{3}\left(\lambda_{1}\right.$ 
$+\lambda_{2}$ in 2$)+0.25 \lambda_{3} ; V($ programme 2$)=\kappa_{1}\left(\lambda_{1}+\lambda_{2} \ln 2\right) ; V($ programme 3$)=$ $\lambda_{1} \lambda_{1}$. Imposing conditions $E$ and $P$ has the effect of making $\lambda_{2}$ and $\lambda_{3}$ both negative. Therefore, under conditions $E$ and $P$ the resulting ranking of the health care programmes is $3>2>1$.

The assessment of the scaling constants follows from a procedure similar to the one outlined at the end of Section 7.2. The only difference is that in this case two trade-off questions have to be asked given that there is one additional scaling constant (one of the $\kappa \mathrm{s}$ ) to assess. The additive value functions $V_{1}$ and $V_{3}$ can be assessed along the lines sketched in Section 3.7 in Keeney and Raiffa (1976).

Condition $\mathrm{TCl}$ is a restrictive condition. Complementarity between $U\left(y_{1}\right)$ and $U\left(y_{2}\right)$ can be introduced by replacing condition $T C I$ by the weaker condition of mutual utility independence between $y_{1}$ and $y_{2}$. If condition $T C I$ is replaced by mutual utility independence, but the other conditions of Theorem 3 still hold. $V(y)$ can be represented by the following equation:

$$
V(y)=\kappa_{1} V_{1}\left[\lambda_{1} U_{1}\left(y_{1}\right)+\lambda_{2} U_{2}\left(y_{2}\right)+\lambda \lambda_{1} \lambda_{2} U_{1}\left(y_{1}\right) U_{2}\left(y_{2}\right)\right]+\kappa_{2} V_{3}\left(y_{3}\right)
$$

This follows from Theorem (6.1) in Keeney and Raiffa (1976). In Eq. (10) three trade-off questions have to be asked to determine all the scaling constants: $\lambda_{1}, \lambda_{2}$ and one of $\kappa_{1}$ and $\kappa_{3}$.

\section{Summary and discussion}

The aim of this paper was to derive equity algorithms for utility-based decision making. Because QALYs are the most commonly encountered utility based measure in the health economics literature, the paper focused on QALY-based decision making. However, it should be emphasized once again that the results of the paper are entirely general: they apply to other utility-based outcome measures as well. Two interpretations of QALYs were considered: QALYs as (vNM) utilities and QALYs as measures of health. In the interpretation of QALYs as utilities, an important issue that has to be resolved is the question whether utilities can be interpersonally compared. The possibility of aggregating utilities over individials has been heavily debated within the economic science. Sections 3 and 4 provided a justification for aggregating QALYs as consistently scaled vNM utilities over individuals.

Section 5 provided a characterization of unweighted aggregation of QALYs. It was shown that two of the conditions underlying the common practice of unweighted aggregation of QALYs over individuals are at variance with two types of equity concerns: a concern for the final distribution of the number of QALYs (ex post equity) and a concern for the fairness of the QALY allocation process (ex ante equity). By relaxing these two conditions, alternative aggregation procedures 
were derived that take into account (some of the) equity considerations. Even though these alternative aggregation procedures are less restrictive than unweighted QALY aggregation, they still impose restrictions on social preferences that may not be tenable in every decision context. Further generalizations of these alternative aggregation procedures are possible. One road to explore is whether results from rank dependent utility theory. currently the most popular alternative for expected utility theory in decision making under risk and uncertainty, can be translated to the context of social decision making. The idea underlying rank dependent utility theory is that different outcomes/individuals get assigned different weights. This is basically the idea underlying the equity principles proposed in this paper.

The paper further shows that incorporating equity concerns can be achieved at relatively low cost: two additional trade-off questions are in general sufficient. Obviously, trade-offs between efficiency and equity considerations are not always easy to make. However, this can be no excuse for not making this trade-off explicit. As shown in this paper, multi-attribute utility theory can be of great help here.

\section{Acknowledgements}

I am grateful to Peter Wakker. Eddy van Doorslaer. Christophe Gonzales, Magnus Johannesson and two anonymous referees for helpful comments on previous drafts of this paper. Any errors ure my own.

\section{Appendix A}

\section{A.1. Prowf of Theorem I}

Condition $H$ is in fact equivalent to the condition of additive independence, which is familiar in multi-atribute utility theory. Additive independence asserts that preferences with respect to lotteries over alternatives depend only on the marginal probability of each outcome occurring and not on the joint probability distribution. Given that it tas been assumed that $z$ : has been defined over a set of (simple) probability distributions, and satisfies the von N:umann Morgenstern axioms. Theorem 11.1 in Fishburn (1970) can be applied (see also Theorem 4 in Fishbum (1965)). According to this theorern.

$$
U(x)=\sum_{i=1}^{n} U_{i}\left(x_{i}\right)
$$

where the $U_{i}\left(x_{i}\right)$. called additive individual utility functions, are defined from the expected utility of the degenerate lottery that gives outcome $x_{i}$ with probability 1 . 
Given the fact that the additive individual utility functions $U_{i}\left(x_{i}\right)$ are unique up to similar positive linear transformations, it follows that the $\lambda_{i} s$ in Harsanyi's theorem are positive. This guarantees positive association between individual and social preferences, one of the conditions from the work of Arrow (1951a).

Imposing condition $A$ on top of the other conditions leads to the QALY utilitarian representation. If $\left(U_{1} U_{2}, \ldots, U_{n}\right)$ is an array of representing additive individual utility functions, then by condition $A$ so are $\left(U_{2}, U_{3}, \ldots, U_{n}\right),\left(U_{3}\right.$, $\left.U_{i}, \ldots, U_{1}, U_{2}\right), \ldots,\left(U_{n}, U_{1}, \ldots, U_{n-1}\right)$. Then $\left\{(1 / n) \sum_{i} U_{i},(1 / n) \sum_{i} U_{i}, \ldots\right.$, $\left.(1 / n) \sum_{i} U_{i}\right\}$ is representing as well and so, by the uniqueness properties of the $U_{i}$, is $\left(\Sigma_{i} U_{i}, \ldots, \Sigma_{i} U_{i}\right)$. This shows that the additive individual utility functions can be chosen identical. Set $U$ equal to one of these additive individual utility functions; this gives the desired result.

Continuity follows from the continuity of $\geq$ and from Theorem 3.2 in Maas and Wakker (1994). Additive independence implies utility independence, which in turn implies independence. The structural assumptions made in Section 5.1 ensure that the other conditions in Maas and Wakker (1994) are fulfilled. Weak order has been assumed. Restricted solvability and the Archimedean axiom follow from the fact that $X_{i}=\mathbb{R}_{+}$and $X=\mathbb{R}_{+}^{n} . \mathbb{R}_{+}$is endowed with the usual Euclidean topology, which is connected and separable. $\mathbb{R}_{+}^{n}$ is endowed with the product topology and, by Theorem 5.3 in Fishburn (1970), is connected and separable. By the proof of Theorem 6.14 in Krantz et al. (1971), continuity of $\geq$ with respect to a connected product topology implies restricted solvability and the Archimedean axiom.

\section{A.2. Proof of Theorem 2}

By Mas and Wakker (1994. Theorem 3.2) conditions $S E$ and $U I 1$ are equivalent to utility independence for all subsets of $I=\{1, \ldots, n\}$ and $U$ is continuous. Then by Theorem 6.1 in Keeney and Raiffa (1976), if $\lambda \neq 0, U(x)$ can be written as

$$
\lambda U(x)+1=\prod_{i=1}^{n}\left[\lambda \lambda_{i} U_{i}\left(x_{i}\right)+1\right]
$$

where $U(x)$ and the $U_{i}$ are scaled between 0 and 1 . Suppose the $U_{i}$ are scaled according to the algorithm proposed by Gafni and Birch (1991). Then a life in full health has utility $l$ for all individuals. If $\lambda=0$, it follows from Eq. (6.12) in Keeney and Raiffa (1976) that $l(x)$ can be written as Eq. (Al), which in combination with condition $A$ gives QALY-utilitarianism.

Suppose without loss of generality that $(0,0, \ldots, 0)$ is the worst social ailocation and set $U(0,0, \ldots, 0)=0$, which is allowed by free scaling of the utility function. By condition $A:(x, 0,0, \ldots, 0) \sim_{z}(0, x, 0, \ldots, 0) \sim \ldots$, $\sim(0,0, \ldots, 0, x)$. Substitute this in Eq. (A2) to give $\lambda \lambda_{1} U_{1}(x)=\lambda \lambda_{2} U_{2}(x)=$ $\ldots=\lambda \lambda_{n} U_{n}(x)$. Thus all $\lambda_{i} U_{i}$ are equal. Set these equal to $U\left(x_{i}\right)$. Rearranging terms gives Eq. (4). 
Denote by $[p, x ;(1-p), y]$ a programme that gives allovation $x$ with probability $p$, and allocation $y$ with probability $(1-p)$. By condition $P,[0.5,(x$, $x, 0, \ldots, 0) ; 0.5,(0,0, \ldots, 0)]$ is preferred to $[0.5,(x, 0, \ldots, 0) ; 0.5,(0, x, 0, \ldots$, 0)]. Calculating the expected utility of these two programmes making use of Eq. (4) gives

$$
\begin{aligned}
& 0.5^{\circ}\left[(1 / \lambda)^{*}(\lambda U(x)+1)^{2}-(1 / \lambda)\right] \\
& >0.5^{\circ}\left[(1 / \lambda)^{*}(\lambda U(x)+1)-(1 / \lambda)\right] \\
& +0.5^{\circ}\left[(1 / \lambda)^{*}(\lambda U(x)+1)-(1 / \lambda)\right]
\end{aligned}
$$

Under the assumption that a QALY is a vNM utility, i.e. $U(x)=x$, Eq. (A3) can be rewritten as

$$
(1 / 2 \lambda)^{\circ}(\lambda x+1)^{2}-(1 / 2 \lambda)>(1 / \lambda)^{*}(\lambda x+1)-(1 / \lambda)
$$

which, after rearranging terms, gives $\lambda x / 2>0$. Given our assumption that $x$ is non-negative and in this particular case cannot equal zero, it foliows that $\lambda>0$.

\section{A.3. Proof of Theorem 3}

Given that $Y$ is assumed to be a Cartesian product, that the one-attribute subsets are intervals in the real numbers. that it is implicitly assumed that the decision maker thinks both attributes of $V(Y)$ should influence social preference (i.e. both atributes are essential), and that $\geq$ on $Y$ is a continuous weak order that satisfies the hexagon condition, by Theorem III.4.1. in Wakker (1989), $V(Y)$ can be represented by

$$
V(y)=\kappa_{1} V_{1}\left(y_{1}^{\text {ce }}\right)+\kappa_{3} V_{3}\left(y_{3}\right)
$$

with $V$ and the additive value functions $V_{i}$ scaled between 0 and 1 , continuous and unique up to similar positive linear transformations. The $\kappa_{i}$ are scaling constants.

Equivalently, Eq. (A5) can be written as

$$
V(y)=\kappa_{1} V_{1}\left[U\left(y_{1}, y_{2}\right)\right]+\kappa_{3} V_{3}\left(y_{3}\right)
$$

Given that condition $\mathrm{TCl}$ is equivalent to additive independence for two attributes, Theorem 2 in Fishburn (1965) can be applied to give

$$
V(y)=\kappa_{1} V_{1}\left[\lambda_{1} U_{1}\left(y_{1}\right)+\lambda_{2} U_{2}\left(y_{2}\right)\right]+\kappa_{3} V_{3}\left(y_{3}\right)
$$

where the $U_{i}$ are scaled between 0 and 1 . unique up to similar positive linear transformations and are continuous given continuity of $V_{l}$.

Finally, it remains to be shown that $y_{i}^{\text {ce }}$ can always be determined. By continuity of the vNM utility function it is possible to find a certainty equivalent for every lottery over $y_{1}$ and $y_{2}$. Furthermore, given continuity of $V_{1} \geq_{A}$ restricted to degenerate probability distributions is continuous. By the vNM 
axioms $z_{A}$ is a weak order. Finally $y_{1}$ and $y_{2}$ are elements of intervals in the real numbers. Thus, by Lemma III.3.3. in Wakker (1989), $\geq_{A}$ satisfies restricted solvability. Suppose $\left(y_{1}, y_{2}\right)$ denotes the certainty equivalent of a lottery. By restricted soivability if $\left(a_{1}, x\right) \succ_{A}\left(y_{1}, y_{2}\right) \succ_{A}\left(c_{1}, x\right)$ where $x$ denotes the value at which the ex post equity index is held fixed, then there exists $\left(b_{1}, x\right)$ such that $\left(b_{1}, x\right) \sim_{A}\left(y_{1}, y_{2}\right)$. If we fix $x$ at the value corresponding to no inequality then there will exist such $\left(a_{1}, x\right)$ and $\left(c_{1}, x\right)$ and thus $y$ can always be deternined.

\section{References}

Arrow, K.J., 1950, A difficulty in the concept of social welfare, Journal of Political Economy 58. 328-346.

Arrow, K.J.. 1951a, Social choice and individual values, 2nd edn, 1963 (Wiley, New York).

Arrow. K.J.. 1951b. Alternative approaches to the theory of choice in risk-taking situations. Econometrica $19.404-437$.

Arkinson, A.B., 1970. The measurement of inequality. Joumal of Economic Theory 2, 244-263.

Ben-Zion and A. Gafni. 1983. Evaluation of public investment in health care: is the risk irrelevant? Journal of Health Economics 2. 161-165.

Blackorby, C.. D. Primont and R.R. Russell. 1978. Duality, separability, and functional structure: Theory and economic applications (Elsevier, New York).

Blau. J.H., 1957. The existence of a social welfare function, Econometrica 25, 302-313.

Broome. J., 1982. Equity in risk bearing. Operations Research 30, 412-414.

Broome. J., 1988. Goodness. faimess and QALYs, in M. Beil and S. Mendus (eds). Philosophy and medical welfare (Cambridge University Press, Cambridge. MA) pp. 57-73.

Broome. J.. 1993. Qalys. Joumal of Public Economics 50. 149-167.

Culyer. A.J., 1989. The normative economics of health care finance and provision. Oxford Review of Economic Policy 5. 34-58.

d'Aspremont. C. and L. Gevers, 1977. Equity and the informational hasis of collective choice, Review of Economic Studies 44, 199-208.

Debreu. G., 1960, Topological methods in cardinal utility theory, in K.J. Arrow, S. Karlin and P. Suppes (eds). Mathematical methods in the social sciences (Stanford University Press, Stanford. CA).

Deschamps, R. and L. Gevers, 1978, Leximin and utilitarian rules: a joint characterization, Journal of Economic Theory 17. 143-16.3.

Diamond. P.A. 1967. Cardinal welfare, individualistic ethics and interpersonal comparisons of utility: comment. Journal of Political Economy 75. 765-766.

Drummond, M.F., G. Stoddart and G.W. Torrance, 1987, Methods for the economic evaluation of health care programs (Oxford University Press. Oxford).

Ellsberg. D., 1954. Classic and current notions of "measurable utility". Economic Joumal 64, 528-556.

Fishburn. P.C.. 1965. Independence in utility theory with whole product sets. Operations Research 13. $28-45$.

Fishbum. P.C.. 1970. Utility theory for decision making (Wiley, New York).

Fishburn. P.C.. 1984. Equity axioms for public risk, Operations Research 32, 901-908.

Fishburn, P.C., 1989, Retrospective on the utility theory of von Neumann and Morgenstern. Journal of Risk and Uncertainty 2. 127-158.

Fishburn. P.C. and P. Straftin. 1989, Equity considerations in public risk evaluation, Operations Research 37, 229-239. 
Fieming. M., 1952. A cardinal concept of welfare, Quarterly Journal of Econonics 66, 366-384.

Gafni, A. and S. Birch. 1991. Equity considerations in utility-based measures of health outcomes in econoric appraisals: an adjustment algorithm. Journal of Health Economics 10, 329-342.

Gasini. A. and G.W. Torrance. 1984. Risk attitude and time preference in health. Management Science 39, 440-45!.

Harris, J., 1988. More and better justice, in M. Bell and S. Mendus (eds). Philosophy and medical welfare (Cambridge University Press, Cambridge. MA) pp. 75-96.

Harsanyi. J.C.. 1955. Cardinal welfare. individualistic ethics, and interpersonal comparisons of utility. Journal of Political Economy 63. 309-321.

Harsanyi. J.C.. 1975. Non-linear social welfare functions, or do welfare economists have a special exemption from Bayesian rationality. Theory Decision 6, 31 1-332.

Harsanyi, J.C., 1987, von Neumann Morgenszem utilities, risk taking and welfare, in G.R. Feiwel (ed.). Arrow and the ascent of modem economic theory (Macmillan. London).

Hildreth. C.. 1953. Alternative conditions for social ordering. Econometrica 21, 81-89.

Jensen. N.E., 1\%67, An iniroduction to Bernoulian utility theory. I. Utility functions, Swedish Journal of Econmmics 69, 163-183.

Keeney. R. and H. Raiffa. 1976. Decisions with Multiple Objectives: Preferences and Value Trade-offs (Wiley. New York).

Keeney, R., 1980. Equity and public risk. Operations Research 28. 527-533.

Krantz. D.H.. R.D. Luce. P. Suppes and A. Tversky. 1971. Foundations of measurement. Vol.1. Additive and polynomial representations (Academic Press. New York).

Lackwom!. M., 1988. Quality of life and resource allocation. in M. Bell and S. Mendus (eds). Philowohy and medical wellare (Cambridge University Press, Cambridge. M.A) pp. 33-55.

Loomes. G. and L. McKenzie. 1989. The use of QALYs in health care decision making. Social Science and Medicine 28, 299-308.

Luce. R.D. and H. Raiffa. 1957. Games and decisions (Wiley. New York).

Maas. A. and P.P. Wakker. 1994. Additive conjoint measurement for multiattribute utility. Journal of Mathematical Psychology 38. 86-101.

Mehrez. A. and A. Gafni. 1989, Quality-adjusted life years, utility theory and healthy-years equivalents. Medical Decision Making 11. 140-146.

Nird. E. 1994. The QALY - a measure of social value rather than individual utility? Health Economics 3. 89-93.

Pliskin. I.S. D.S. Shepard and M.C. Weinstein. 1980. Utility functions for life years and health status. Operaioions Revearch $28.206-224$.

Savage. L.J., 1954. The foundations of statistics (Wiley, New York).

Sen. A.K.. 1970, Collective choice and social Weltare (Holden Day, San Francisco, CA).

Sen. A.K.. 1973. On economic inequality (Clarendon. Oxford).

Sen. A.K.. 1976. Welfare inequalities and Rawlsian axiomatics. Theory Decision 7. 243-262.

Sen. A.K., 1977, On weights and measures: informational constraints in social welfare analysis, Econometrica 45, 1539-1572.

Sen. A.K.. 1979. Personal utilities and public judgements: or what's wrong with welfare economics?. Economic Joumal 89. 537-558.

Smith. A., 1987. Qualms about QALYs, Lancet i. 1134.

Torrance. G.W.. 1986. Measurement of health state utilities for economic appraisal: a review, Journal of Health Economics $5.1-30$.

Tormance, G.W. and D. Feeny. 1989. Utilities and quality-adjusted life years, International Journal of Techrology Assessment in Heath Care 5, 559-575.

Ulph. A.. 1982. The role of ex ante and ex post decisions in the valuation of life. Joumal of Public Ecomonics 18. 265-276.

Wagstaff. A.. 1991. QALYs and the equity-efficiency trade-off. Journal of Health Economics 10. $21-41$. 
Wakker, P.P., 1989, Additive representations of preferences: a new foundation of decision analysis (Kluwer. Dordrecht).

Wakker, P.P.. Separating marginal utility and risk aversion. Theory Decision 36, 1-44.

Williams. A., 1981. Welfare economics and health status measurement, in J. van der Gaag and $M$. Perlman (eds). Health, economics and health economics (North-Holland. Amsterdam) pp. 271-281.

Williams. A.. 1993. Priorities and research strategy in health economies for the 1990s. Health Economics 2. 295-302. 